\title{
Giant solitary trichoepithelioma with desmoplasia on histopathology: An unusual finding
}

\section{Sundeep Chowdhry1, Shikha Gupta', Anand K Verma², Jagbir Singh', Paschal D’souza', Manish S Jain ${ }^{3}$}

${ }^{1}$ Department of Dermatology, ESI PGIMSR, Basaidarapur, Ring Road, New Delhi-110015, India, ${ }^{2}$ Department of Pathology, ESI PGIMSR, Basaidarapur, Ring Road, New Delhi-110015, India, ${ }^{3}$ Department of Plastic Surgery, ESI PGIMSR,

Basaidarapur, Ring Road, New Delhi-110015, India

Corresponding author: Dr Shikha Gupta, E-mail: dr_shikhaarora@yahoo.co.in

\begin{abstract}
Trichoepithelioma is a well-recognized hamartoma of hair germ clinically presenting as solitary or multiple papules. Uncommonly, it may present as a large solitary lesion, termed as giant solitary trichoepithelioma (GST). A 50-year old man presented with a large, pedunculated mass over the root of nose obstructing visual field. It was excised and sent for histopathological examination. H\&E staining revealed in addition to characteristic features of trichoepithelioma, a desmoplastic hypocellular stroma. After taking into account the clinical and histopathological features, a diagnosis of GST was made. This case is the first case to the best of authors' knowledge describing desmoplastic stroma in a case of GST. It is also noteworthy because it reports the largest GST described so far over the nose.
\end{abstract}

Key words: Desmoplasia; Giant solitary trichoepithelioma; Trichoepithelioma

\section{INTRODUCTION}

Giant solitary trichoepithelioma (GST) is an uncommon variant of trichoepithelioma which usually occurs on thigh and perianal region and measures several centimetres in diameter [1]. Only 18 cases of giant solitary trichoepithelioma (GST) have been described so far across the world, the largest measuring $17 \times$ $8 \mathrm{~cm}$ [2]. Four of these have been reported to occur in the nasal area, the largest of which measured $3 \times$ $2.5 \mathrm{~cm} \mathrm{[3].}$

The histopathology is similar to classical trichoepithelioma, which is characterized by horn cysts and aggregates of basaloid cells in superficial dermis. The stroma surrounding these aggregates characteristically has moderate number of fibroblasts[1]. However, hypocellular desmoplastic stroma, a unique feature of the desmoplastic variant of trichoepithelioma (DTE), has not yet been described in GST, to the best of the authors' knowledge.
The authors describe here a case of GST, the largest reported so far in the nasal region with a histological feature of desmoplastic, hypocellular stroma, which initially led to diagnostic dilemma.

\section{CASE REPORT}

A 50 year old man presented with a mass over the root of nose for past five years. It was insidious in onset, asymptomatic, initially of pea-size and gradually increased to reach the size of a tennis ball. Initially unperturbed, the patient sought medical advice when the swelling caused obstruction of vision owing to its large size. There was no history of trauma at the site. Past history and family history were unremarkable.

On examination, a well-defined pedunculated mass of size $10 \times 8 \times 6 \mathrm{~cm}$ was seen arising from the root of nose adjacent to right medial canthus having a pedicle measuring $3 \times 2 \mathrm{~cm}$ (Fig. 1). The surface was 
irregular with few erosions, areas of depigmentation, hyperpigmentation, crusting and atrophic scarring. On palpation, it was non-tender, firm in consistency and on manipulation, expressed scanty serous discharge. The swelling was attached to the overlying skin though not fixed to underlying structures. There was no significant lymphadenopathy. The visual acuity, slit lamp \& fundoscopic examinations were within normal limits, however due to its massive size, the lesion obstructed most of the visual field of the right eye and simultaneously resulted in deviation of the nasal tip to left side.

The differential diagnoses considered were basal cell carcinoma, large acrochordon, squamous cell carcinoma and solitary giant trichoepithelioma.

The lesion was excised under local anaesthesia and grafting was performed with donor skin taken from right thigh. The excised specimen was sent for histopathological examination. The H\&E staining of the non- ulcerated base of the lesion revealed a benign adnexal tumor with uniform basaloid cells arranged in islands as well as in narrow strands (Fig. 2a). There was continuity with epidermis at few places. The surrounding stroma was characteristically desmoplastic showing hypocellular tissue with fibrosis (Fig. 2b). There was homogenously eosinophilic collagen along with mild inflammatory infiltrate in superficial and mid dermis composed predominantly of lymphocytes. Pilar differentiation with some abortive hair follicles was seen (Fig. 2c). Also several horn cysts were present. Foreign body giant cell reaction was appreciable at few sites (Fig. 2b). The margins were tumor free. Immunohistochemical

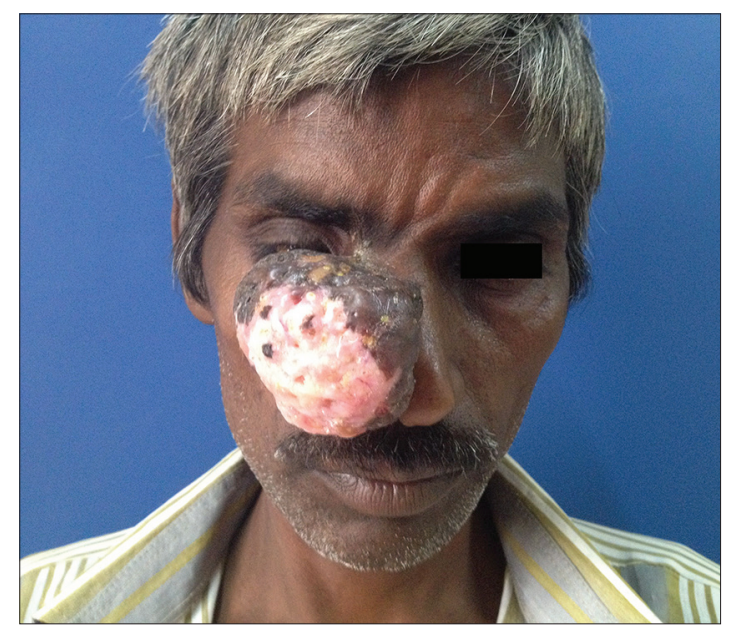

Figure 1: A large mass arising from the root of the nose obstructing the visual field.
(IHC) markers cytokeratin (CK) 5/6 and CK 8 showed positive results with focal and weakly positive staining for Ber-EP4 and CK 20. Thus a histopathological diagnosis of trichoepithelioma with desmoplastic features was made.

The post-operative period was uneventful and the graft was well taken up (Fig. 3). There has been no recurrence one year after the surgery.

Prior to the study, patient gave written consent to the examination and biopsy after having been informed about the procedure.

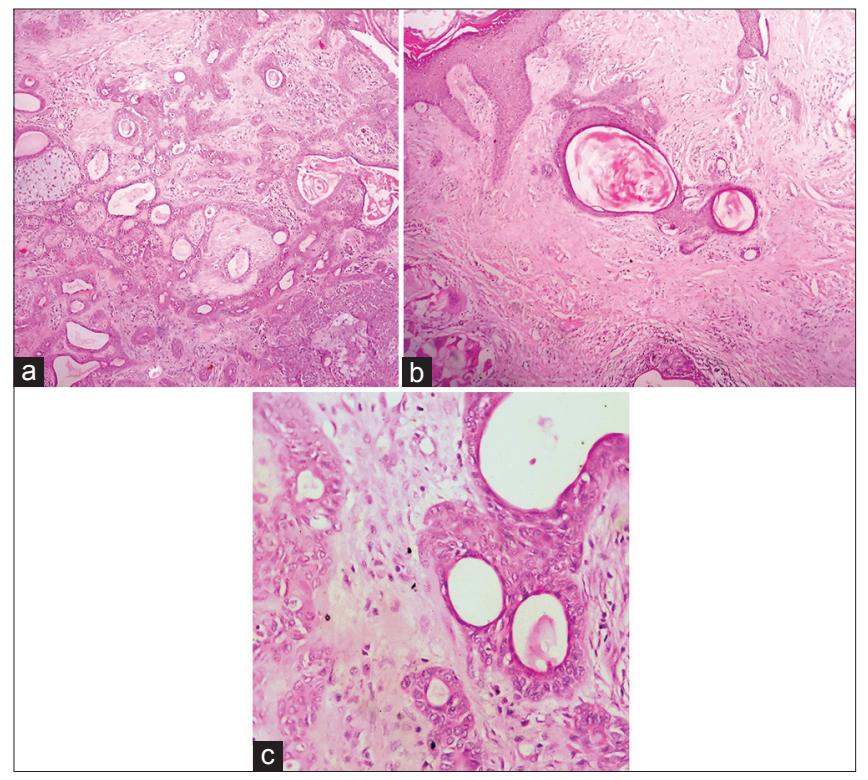

Figure 2: (a) Rare cellular areas showing basaloid cells arranged in islands and narrow strands (H\&E 40x), (b) Marked desmoplasia, horn cysts, foreign body giant cell reaction seen in dermis. Focal chronic inflammatory infiltrate also seen (H\&E 100x), (c) Basaloid cells in strands and forming abortive hair follicles at few places (H\&E 400x).

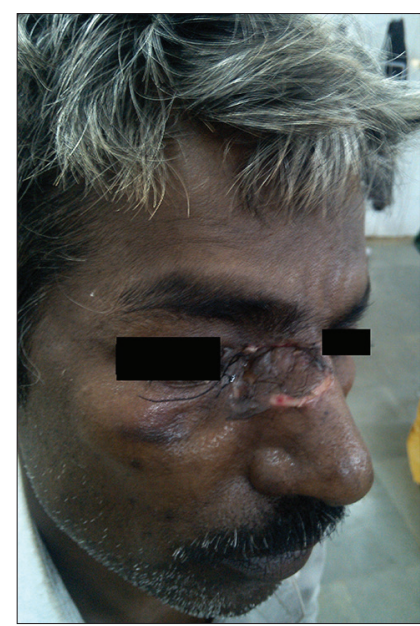

Figure 3: Well taken-up graft post excision. 


\section{DISCUSSION}

Trichoepithelioma is a hamartoma of the hair germ which is said to represent the more mature end of the spectrum of trichoblastoma. It may present as either multiple lesions or a solitary nodule [4]. GST has been described as a rare variant of trichoepithelioma by some authors [1], while others classify it as a variant of trichoblastoma [4].

The histopathology of GST shows features of classical trichoepithelioma [4] which is characterized by islands of uniform basaloid cells with small keratinous cysts lined by stratified squamous epithelium. The stroma is prominent and loosely arranged. Epithelial structures resembling hair papillae or abortive hair follicles may be seen. Rupture of keratinous cysts with extrusion of keratinous debris may result in a small foreign- body granulomatous reaction in stroma, which was also observed in the present case. Immunohistochemical staining of trichoepithelioma reveals strong reactions for cytokeratins (CK) 5/6 and CK8. Ber-EP4 is positive focally in most trichoepitheliomas, contrasting with the usual diffuse staining in basal cell carcinoma [5].

On the other hand, DTE represents a distinct clinicopathological entity and is characterized clinically by a 3-8 $\mathrm{mm}$, annular indurated lesion on face with depressed nonulcerated centre and histologically by a triad of narrow epithelial strands, horn cysts and desmoplastic stroma $[1,4]$.

The management of GST includes excision of the lesion to confirm the diagnosis as well as to allay the discomfort associated with its large size. Local recurrence is exceptional [4]. Surgical excision with grafting was performed successfully in this patient.

It is imperative here to discuss the causes of 'desmoplasia' which is a process of fibrosis induced by activation of fibroblasts [6]. Desmoplastic changes have been reported to occur following inflammation [7], other types of tissue injury and neoplasm [6]. Inflammation is commonly associated with the multiple familial variant of trichoepithelioma, particularly the ones caused by CYLD gene mutations [8,9].

In the present case, it may be hypothesized that inflammation was in part responsible for the desmoplastic changes occurring in the stroma. The cause of the inflammation could be recurrent infections secondary to the surface erosions. However, it is difficult to conclusively comment on the same.

\section{CONCLUSION}

This case is being reported due to the unusually large size (the largest described so far over the nose and the $2^{\text {nd }}$ largest GST described overall) of nasal trichoepithelioma as well as the histological evidence of hypocellular fibrotic stroma in a lesion clinically appearing as a GST. This finding has not been described previously in the literature which prompted the authors to report this case.

\section{CONSENT}

The examination of the patient was conducted according to the Declaration of Helsinki principles.

\section{REFERENCES}

1. Ahmed TSS, Priore JD, Seykora JT. Tumors of the Epidermal Appendages. In: Lever's Histopathology of the Skin (Elder DE, Elenitsas R, Johnson BL, Murphy GF, Xu G, eds), $10^{\text {th }}$ edn. Philadelphia: Lippincott Williams \& Williams. 2008; 857-861.

2. Oursin C, Krüger HJ, Sigmund G, Hellerich U. [MR imaging of a giant solitary trichoepithelioma of the skin]. [Article in German]. Radiologe. 1991;31:574-6. [as quoted in: Goyal S, Mahajan NC, Garg M, Goyal S. Giant Solitary Nodular Trichoepithelioma: A Case Report and Review of Literature. Arch Clin Exp Surg. 2012;1:58-60.]

3. Patrocinio LG, Damasceno PG, Patrocinio TG, Patrocinio JA. Solitary nasal trichoepithelioma. Braz J Otorhinolaryngol. 2008; 74:637.

4. Calonje E. Tumours of the Skin Appendages. In: Rook's Textbook of Dermatology (Burns T, Breathnach S, Cox N, Griffiths C, eds), $8^{\text {th }}$ edn. Oxford: Wiley-Blackwell. 2010; 53.8-53.10.

5. Weedon's Skin Pathology (Weedon D, ed). Chapter 33, Tumors of Cutaneous Appendages, $3^{\text {rd }}$ edn. London: Churchill Livingstone-Elsevier. 2010; 760-762.

6. Ohtani H. Pathophysiologic significance of host reactions in human cancer tissue: desmoplasia and tumor immunity. Tohoku J Exp Med. 1999;187:193-202.

7. Stramer BM, Mori R, Martin P. The inflammation-fibrosis link? A Jekyll and Hyde role for blood cells during wound repair. J Invest Dermatol. 2007;127:1009-17.

8. Blake PW, Toro JR. Update of cylindromatosis gene (CYLD) mutations in Brooke-Spiegler syndrome: novel insights into the role of deubiquitination in cell signaling. Hum Mutat. 2009;30:1025-36.

9. Gray HR, Helwig EB. Epithelioma Adenoides Cysticum and Solitary Trichoepithelioma. Arch Dermatol. 1963;87:102-14.

Copyright by Sundeep Chowdhry, et al. This is an open access article distributed under the terms of the Creative Commons Attribution License, which permits unrestricted use, distribution, and reproduction in any medium, provided the original author and source are credited.

Source of Support: Nil, Conflict of Interest: None declared. 